\title{
Trimetazidine improves right ventricular function by increasing miR-21 expression
}

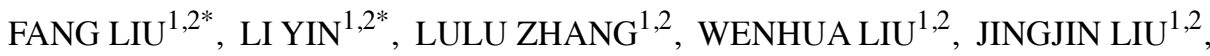 \\ YONGSHUN WANG ${ }^{1,2}$ and BO YU $^{1,2}$ \\ ${ }^{1}$ Key Laboratories of Education Ministry for Myocardial Ischemia Mechanism and Treatment, ${ }^{2}$ Department of Cardiology,
Second Affiliated Hospital of Harbin Medical University, Harbin, Heilongjiang, P.R. China
}

Received April 11, 2012; Accepted June 7, 2012

DOI: $10.3892 /$ ijmm.2012.1078

\begin{abstract}
Trimetazidine (TMZ) improves left ventricular (LV) function and alleviates angina. TMZ is a metabolism-related drug, but its molecular actions and non-metabolic effects have not yet been elucidated. In this study, we investigated whether TMZ improves right ventricular (RV) function and decreases apoptosis in RV myocardial cells (RVMCs) by regulating miRNA-21 (miR-21) expression in vitro and in vivo. We used cultivated RVMCs and LV myocardial cells (LVMCs) and provided hypoxic stimulation for $24 \mathrm{~h}$ to induce $\mathrm{MC}$ apoptosis. RVMCs showed more severe apoptosis as indicated by terminal deoxynucleotidyl-transferase-mediated dUTP nick end-labeling (TUNEL) staining and caspase-3 protein and activity assays. The decrease in miR-21 expression was more dramatic in RVMCs. Subsequently, TMZ $(10 \mu \mathrm{M})$ was added to the RVMCs prior to hypoxic stimulation. The TMZ-treated RVMCs showed less apoptosis and an increased expression of miR-21. The transfection of RVMCs with a miR-21-specific inhibitor weakened the protective effects of TMZ. To evaluate TMZ effectiveness in right heart failure, we used a combination treatment of hypoxia and the vascular endothelial growth factor receptor blocker, Su5416, to construct a stable model, and administered TMZ. TMZ improved RV function (as indicated by an increase in tricuspid annular plane systolic excursion), and inhibited fibrosis. TMZ also protects RVMCs againts apoptosis and increases miR-21 expression.
\end{abstract}

\section{Introduction}

Right ventricular (RV) function may be impaired in pulmonary hypertension $(\mathrm{PH})$, congenital heart disease (CHD), and

Correspondence to: Dr Bo Yu, Department of Cardiology, Second Affiliated Hospital of Harbin Medical University, 148 BaoJian Road, Harbin, Heilongjiang 150086, P.R. China

E-mail: yubodr@163.com

${ }^{*}$ Contributed equally

Key words: apoptosis, right ventricular failure, miRNA-21, trimetazidine coronary artery disease (CAD) and in patients with left-sided heart failure or valvular heart disease. The right ventricle (RV) and left ventricle (LV) of the mammalian heart originate from different progenitor cells during cardiac morphogenesis $(1,2)$ and undertake distinct functions in the circulation $(3,4)$. Animal studies have also demonstrated that the RV and LV exhibit distinct responses to neurohormone stimulation and hypoxia $(5,6)$. Current therapies on RV dysfunctions focus mainly on protopathy, with little attention being paid to the RV. Hence, the evidence that guides the management of isolated $\mathrm{RV}$ failure is not nearly as well established as the evidence that guides the management of chronic heart failure resulting from LV dysfunction. Hence, investigating the mechanism of right heart failure and choosing therapy is important.

Trimetazidine (TMZ) is a first-line anti-anginal agent. The mechanism of action of TMZ can be attributed to the optimization of energy metabolism. A number of clinical studies have shown the efficacy of TMZ in the treatment of various forms of ischemia, including angina pectoris $(7,8)$ and acute coronary syndromes (9). These beneficial effects have been attributed to the inhibition of fatty acid oxidation by TMZ. The mechanism of action of TMZ is believed to be a based on metabolic changes; however, its molecular opus moderandi and non-metabolic effects remain to be elucidated. Liu et al showed that TMZ effectively inhibited myocardial fibrosis (10). In vitro evidence suggests that TMZ can also modulate a transition in mitochondrial permeability and protect myocardial cells (MCs) against ischemia-reperfusion injury $(11,12)$. Whether TMZ can protect MCs in the RV against hypoxic injury is not known.

miRNAs are small, non-coding RNAs of 18-25 nt that regulate gene expression by forming base-pair interactions with the 3' untranslated regions (3'UTR) of target genes, thereby suppressing target mRNA stability or translation into proteins (13). Previous studies have shown that miRNAs play particularly important roles in the development of the cardiovascular system and cardiovascular disease in humans (14). miRNA-21 (miR-21) is highly expressed in the cardiovascular system and has been linked to many forms of heart disease $(15,16)$. Furthermore, one of the most important functions of miR-21 is its anti-apoptotic role. Evidence has shown that miR-21 can protect MCs from hypoxia and $\mathrm{H}_{2} \mathrm{O}_{2}$-induced apoptosis via targeting Fas ligand (FasL) and programmed cell death 4 (PDCD4) (17,18). 
In the present study, we investigated the protective role of TMZ in right heart failure, and ascertained whether the protective role of TMZ is associated with regulation of miR-21 expression.

\section{Materials and methods}

The study protocol was approved by the Ethics Committee of the Second Affiliated Hospital of Harbin Medical University (Harbin, China). All animal procedures were carried out in accordance with the guidelines specified by the Johns Hopkins Animal Care and Use Committee.

Cell culture and treatment. Hearts were isolated from 1- or 2-day-old Sprague-Dawley (SD) rats and separated into the right and left heart plus atrioventricular septum using a microscope. The chambers were separated by first removing the left and right atria from the base of the heart. The RV was then dissected from the LV and septum by inserting iridectomy scissors into the opening of the tricuspid valve and cutting around the interface of the RV and septum, leaving a little 'rim' of RV tissue at the margins, then removing excess tissue around the LV and septum. This procedure resulted in an oblong portion of the RV and a globular section of LV and septum. MCs from the LV and RV were isolated and cultured according to a wellestablished method. Briefly, the 2 ventricles were minced and trypsinized at $37^{\circ} \mathrm{C}$. Cells were centrifuged ( $800 \mathrm{x} \mathrm{g}$ for $\left.5 \mathrm{~min}\right)$ and resuspended in Dulbecco's modified Eagle's medium/F-12 (Gibco, Billings, MT, USA) supplemented with $10 \%$ fetal bovine serum (FBS; HyClone-Thermo Scientific, Jülich, Germany), $100 \mathrm{U} / \mathrm{ml}$ penicillin and $100 \mu \mathrm{g} / \mathrm{ml}$ streptomycin. After cell panning at $37^{\circ} \mathrm{C}$ for $90 \mathrm{~min}$, unattached MCs were harvested and seeded at a density of $5 \times 10^{4}$ cells $/ \mathrm{cm}^{2} .5$-Bromo2'-deoxyuridine (BrdU; $0.1 \mathrm{mM}$; Sigma-Aldrich, St. Louis, MO, USA) was added to the culture for $36 \mathrm{~h}$ to inhibit the proliferation of non-MCs. To ensure that the following experiment involved the same number of MCs, we also used flow cytometry to count MCs and non-MCs. The number of MCs in the RV and LV were identical $(95.77 \pm 0.012$ and 95.57 \pm 0.007 , respectively; $\mathrm{P}>0.05$, data not shown). The MCs were then treated with $10 \mu \mathrm{M}$ TMZ.

Animal model of right heart failure. A model of right heart failure was created as described previously (19). Male SD rats (150-200 g) received a single subcutaneious (s.c.) injection of the vascular endothelial growth factor receptor (VEGFR) blocker, Su5416 $(20 \mathrm{mg} / \mathrm{kg})$. The rats were then placed in a normobaric hypoxia $\left(10 \% \mathrm{O}_{2}\right)$ chamber for 4 weeks in order to cause $\mathrm{RV}$ failure (SuHx group), whereas the control rats (CON group) were reared in room air for the same period of time. The SuHx group was randomly subdivided into 2 groups: 1 group received TMZ (10 mg/kg/day, SuHx + TMZ group) and the other group (SuHx group) received placebo treatment. Animals were sacrificed 4 weeks later after echocardiographic assessment. All animals were anesthetized with an intraperitoneal (i.p.) injection of sodium pentobarbital (130 $\mathrm{mg} / \mathrm{kg}$ ) prior to removing the heart. RV hypertrophy was created by separating the RV from the LV plus septum $(\mathrm{LV}+\mathrm{S})$, weighing these components, and calculating the ratio of $\mathrm{RV} /(\mathrm{LV}+\mathrm{S})$.
Isolation of $M C s$ in the $R V$ from rats. The adult rat ventricle $\mathrm{MC}$ isolation was carried out using an adaptation of the method described previously (20). The hearts were removed quickly from the rats and enzymatically dissociated. Rectangular, trypan blue-excluding cells constituted approximately $80 \%$ of all myocytes. The myocytes were plated into the 6-well plates coated with $0.5 \mu \mathrm{g} / \mathrm{cm}^{2}$ of laminin (Sigma), at a density of $2 \times 10^{4}$ cells $/ \mathrm{cm}^{2}$. The cultures were incubated in serum-free medium (SFM). The SFM was changed 30 min after plating to remove the myocytes that had not attached to the dish. All the myocytes were cultivated for $24 \mathrm{~h}$ before the experiments.

Echocardiographic assessment. Echocardiographic measurements were taken of the inner diameter of the RV and tricuspid annular plane systolic excursion (TAPSE) as previously reported (21).

Histological measurements. Histological examinations were performed in 3 groups of 5 rats that were submitted to the same exposure protocol. After sacrifice, each heart was immediately dissected, and the RV and LV were placed in a formalin solution, and cut into slices (40 $\mu \mathrm{m}$, long axis) for Masson staining. Structural changes were evaluated qualitatively by an investigator blinded to the treatment protocol.

Quantitative reverse transcription-polymerase chain reaction (RT-PCR). miR-21 was detected by qRT-PCR as described previously (19). Briefly, total RNA was harvested from the cells and reverse-transcribed into cDNA. For miR-21, a miScript Reverse Transcription kit and miScript SYBR-Green PCR kit were used (Qiagen, Hilden, Germany). As the internal control, U6 was used for the normalization of the miR-21 templates. The threshold cycle $(\mathrm{Ct})$ was set within the exponential phase of the PCR. Relative gene expression was calculated by comparing cycle times for each target PCR. The target PCR Ct values were normalized by subtracting the U6 $\mathrm{Ct}$ value, which provided the $\Delta \mathrm{Ct}$ value.

Transient transfection. miR-21 inhibitors were synthesized as unconjugated and fully phosphorothioate mixed DNA oligonuleotides with a 6-carboxyfluorescein (FAM) moiety at the 5' terminus (Shanghai GenePharma Co., Shanghai, China). Cells were transfected with $2 \mu \mathrm{g}$ inhibitor in 6-well plates using a commercial transfection reagent (X-tremeGene siRNA Transfection Reagent; Roche, Basel, Switzerland) according to the manufacturer's instructions.

Western blot analysis. For western blot analyses, $60 \mathrm{~g}$ of total protein were extracted from the harvested cells. Samples were loaded onto $12 \%$ sodium dodecyl sulfate-polyacrylamide gel electrophoresis (SDS-PAGE) gels. These were separated by electrophoresis, and transferred onto Immobilon-P polyvinylidene fluoride nylon membranes. Membranes were incubated with antibodies against caspase-3 (Cell Signaling Technology, Inc., Danvers, MA, USA), and $\beta$-actin (Santa Cruz Biotechnology, Inc., Santa Cruz, CA, USA).

Detection of caspase-3 activity. Caspase-3 activity was measured using a commercial spectroscopic assay based on the ability of caspase-3 to convert acetyl-Asp-Glu-Val-Asp 
A
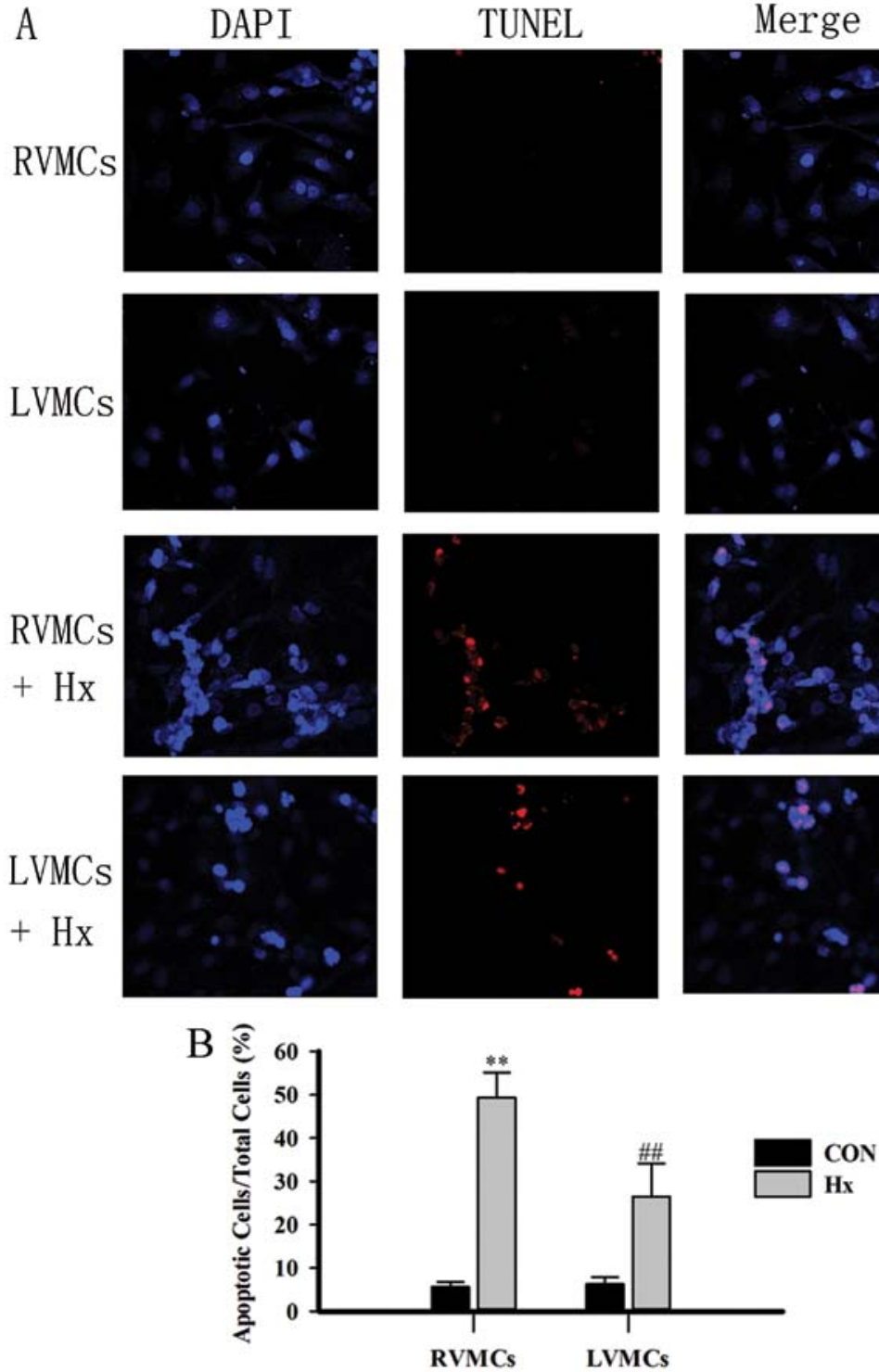
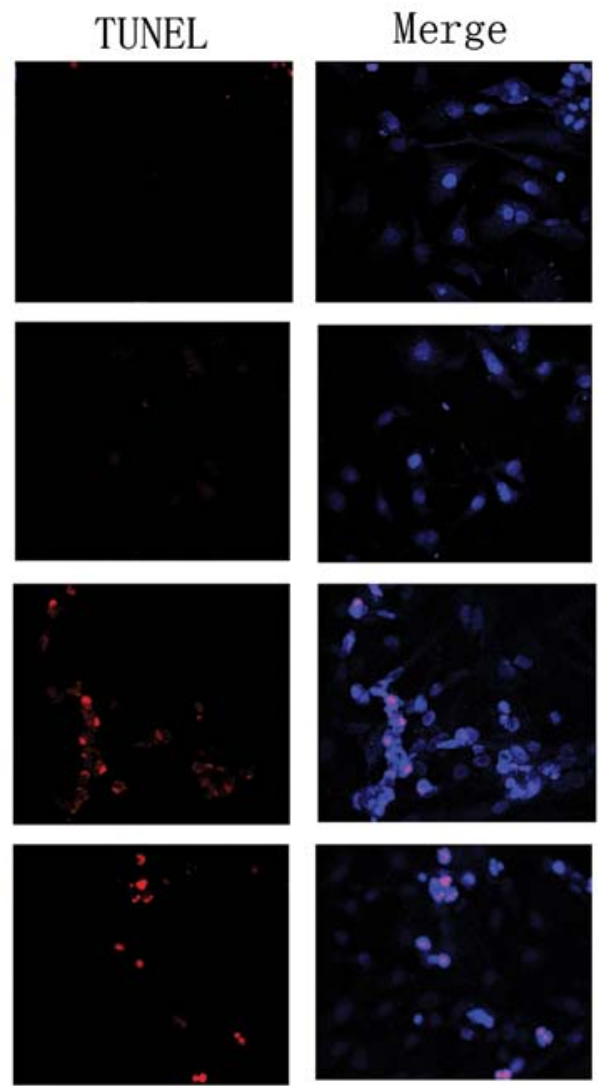

Figure 1. TUNEL staining for the evaluation of hypoxia-induced apoptosis in RVMCs and LVMCs. (A) Fluorometric TUNEL analyses of apoptotic cells in RVMCs and LVMCs after $24 \mathrm{~h}$ of hypoxia. TUNEL ${ }^{+}$cells are indicated by red fluorescence. (B) Quantification of TUNEL ${ }^{+}$cells. Data are expressed as percentages. ${ }^{* *} \mathrm{P}<0.01$ compared with RVMCs, and ${ }^{\# \#} \mathrm{P}<0.01$ compared with LVMCs.

p-nitroanilide (Ac-DEVD-pNA) into a yellow formazan product, $\mathrm{p}$-nitroaniline (pNA). The lysates were centrifuged at $12,000 \mathrm{x} \mathrm{g}$ for $10 \mathrm{~min}$ at $37^{\circ} \mathrm{C}$, and the supernatants incubated in 96-well microtiter plates with $20 \mathrm{ng}$ Ac-DEVD-pNA for $4 \mathrm{~h}$ at $37^{\circ} \mathrm{C}$. The optical density (OD) at an absorbance of $405 \mathrm{~nm}$ $\left(\mathrm{OD}_{405}\right)$, which is indicative of caspase-3 activity, was read on a 96-well plate reader (Infinite M200; Tecan, Männedorf, Switzerland). Each assay was performed in triplicate.

Terminal deoxynucleotidyl-transferase-mediated dUTP nick end-labeling (TUNEL) assay. TUNEL staining was carried out using an In Situ Cell Death Detection kit (Roche) according to the manufacturer's instructions. RVMCs and LVMCs cultured on coverslips in 24-well plates were fixed in $4 \%$ paraformaldehyde and stained. TUNEL-positive cells were imaged under a confocal laser scanning microscope (Fluo View v5.0 FV300; Olympus, Tokyo, Japan) and counted in 10 randomly chosen fields. The results were expressed as the percentage of TUNEL-positive cells relative to total number of cells counted. 4',6-Diamidino-2-phenylindole dihydrochloride DAPI ( $1 \mu \mathrm{g} / \mathrm{ml}$; Sigma-Aldrich) was used for nuclear counterstaining.

Statistical analyses. Data are the means \pm standard error (SE). Group comparisons were made using an analysis of variance (ANOVA) with Fisher's test. $\mathrm{P}<0.01$ or $\mathrm{P}<0.05$ were considered to indicated statistically significant differences.

\section{Results}

To compare apoptosis between RVMCs and LVMCs after hypoxia, we used separate cultures of neonatal rat MCs from the RV and LV (see Materials and methods). TUNEL staining was used to evaluate apoptosis in the MCs. The RVMCs and LVMCs exhibited a significantly greater proportion of TUNEL-positive nuclei compared with those from the control group (Fig. 1A and B). In addition, there were slightly more RVMCs than LVMCs. The caspase-3 protein assay and 

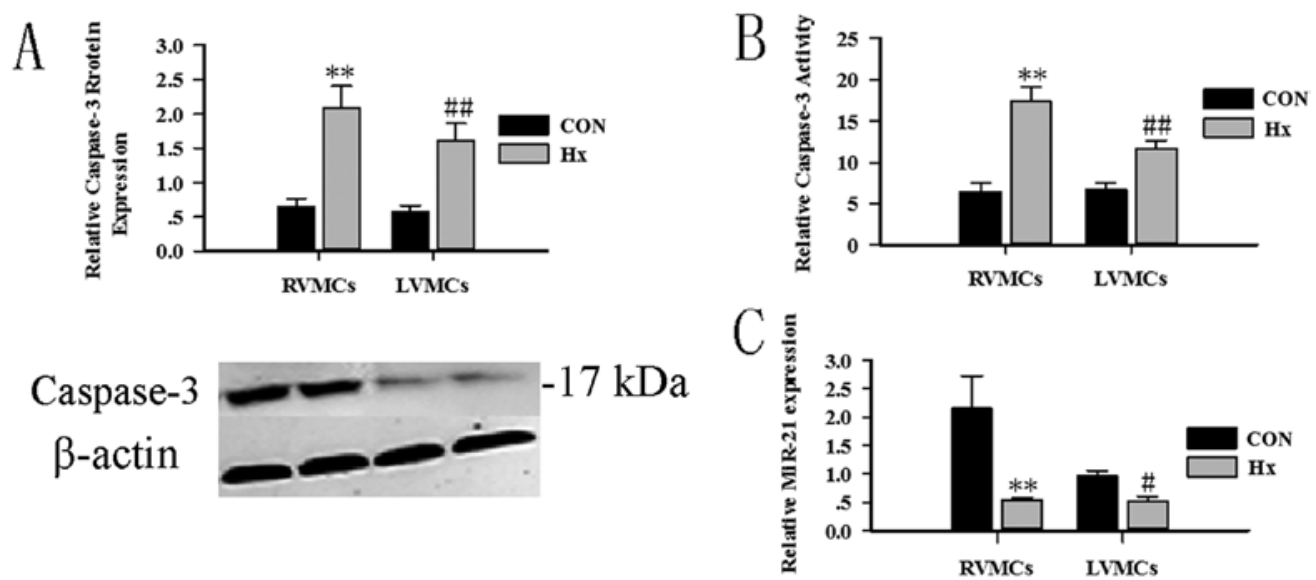

Figure 2. Apoptosis of MCs was accompanied by the decreased expression of miR-21. (A) Representative western blot analyses and average densitometric data showing that hypoxia increased the amount of the 17-kDa (active) cleavage product of caspase-3 in RVMCs and LVMCs. (B) Relative caspase-3 activity was calculated as the fluorescence intensity ratio of the staining of hypoxia-treated cells to the staining of control cells. Results are the means \pm SEM from 4 independent experiments for each treatment protocol. RVMCs and LVMCs showed increased caspase-3 activity. (C) Relative expression of miR-21 before and after hypoxia stimulation. The expression of miR-21 relative to U6 RNA was determined using the $2^{-\Delta \Delta C t}$ method. Results are the means of 4 treatments for each group. The decrease in miR-21 expression in RVMCs was more dramatic than that in LVMCs. ${ }^{* *} \mathrm{P}<0.01$ compared with the control group of RVMCs; ${ }^{\# \#} \mathrm{P}<0.01$ compared with the control group of LVMCs.

Table I. Rat characteristics, ultrasound findings in control rats, 6 weeks after Su5416 treatment and hypoxia, and 6 weeks after Su5416 treatment, hypoxia and TMZ treatment.

\begin{tabular}{lllllc}
\hline & BW $(\mathrm{g})$ & RV $(\mathrm{mg})$ & $\mathrm{RV}(\mathrm{cm})$ & $\mathrm{RV} / \mathrm{LV}+\mathrm{S}$ & TAPSE $(\mathrm{mm})$ \\
\hline CON $(\mathrm{n}=6)$ & $380 \pm 15$ & $230 \pm 18$ & $1.82 \pm 0.45$ & $0.36 \pm 0.05$ & $3.5 \pm 0.2$ \\
SuHx + PBS (n=8) & $317 \pm 24^{\mathrm{a}}$ & $324 \pm 36^{\mathrm{a}}$ & $3.05 \pm 0.47^{\mathrm{a}}$ & $0.60 \pm 0.1^{\mathrm{a}}$ & $1.6 \pm 0.5^{\mathrm{a}}$ \\
SuHx + TMZ (n=8) & $352 \pm 19^{\mathrm{b}}$ & $287 \pm 47^{\mathrm{b}}$ & $2.6 \pm 0.34^{\mathrm{b}}$ & $0.51 \pm 0.07^{\mathrm{b}}$ & $2.6 \pm 0.7^{\mathrm{b}}$ \\
\hline
\end{tabular}

BW, body weight; RV (mg), RV weight; RV (cm), RV inner diameter; LV, LV weight; S, septal weight; TAPSE, tricuspid annular plane systolic excursion. Values are the means $\pm \mathrm{SD}$. ${ }^{\mathrm{a}} \mathrm{P}<0.05$ vs. $\mathrm{CON}$; ${ }^{\mathrm{b}} \mathrm{P}<0.05$ vs. $\mathrm{SuHx}+\mathrm{PBS}$.

activity assay were used to evaluate MC apoptosis (Fig. 2A and B). miR-21 expression was evaluated at the same time. Twenty-four hours after hypoxia treatment, RVMCs and LVMCs showed a decreased expression of miR-21 (Fig. 2C).

We then investigated whether TMZ can protect RVMCs from hypoxia-induced injury: the RVMCs were treated with $10 \mu \mathrm{M}$ TMZ prior to hypoxia treatment. The TUNEL assay (Fig. 3A and B) showed that TMZ decreased the number of TUNEL-positive nuclei. The TMZ-treated RVMCs exhibited a reduced expression of caspase- 3 protein and reduced activity (Fig. 3C and D). We then detected anti-apoptotic miRNA and miR-21 expression with RT-PCR. The TMZ-treated RVMCs demonstrated an increased expression of miR-21 (Fig. 3E). To discover whether the increases in miR-21 expression were associated with the protective role of TMZ, we used a synthesized miR-21-specific inhibitor (Shanghai GenePharma Co) to decrease miR-21 expression. This miR-21 inhibitor decreased miR-21 expression by almost 50\% (Fig. 3F). We then investigated the protective role of TMZ again using TUNEL staining, the caspase- 3 protein assay and caspase- 3 activity assay. The amount of caspase -3 protein and the activity of caspase- 3 decreased following miR-21 inhibition (Fig. 3C and D). TUNEL staining also showed that this miR-21 inhibitor weakened the protective role of TMZ (Fig. 3A and B).
Our results demonstrate (at least in part) that TMZ can protect RVMCs against hypoxic injury by regulating miR-21 expression on a cellular level. We then wished to elucidate whether TMZ can be effective in an animal model of right heart failure.

In this study, we used a stable model of right heart failure by employing combination treatment with Su5416 and hypoxia. Table I shows the characteristics of all 3 groups of rats. The SuHx group showed a dramatic decrease in TAPSE and dilation of the inner diameter of the RV. TMZ produced a slight increase in TAPSE, and decreased the size of the $\mathrm{RV}$, indicating that TMZ improves RV function. Fibrosis is another important feature of RV function. The TMZ-treated group exhibited a low level of fibrosis (Fig. 4A). Furthermore, the amount of caspase- 3 protein, the activity of caspase- 3 , and miR-21 expression are indices of right heart function. miR-21 is highly expressed in MCs and fibroblasts. Hence, we isolated RVMCs and then detected the amount of caspase- 3 protein, the activity of caspase- 3 and miR-21 expression. The SuHx group showed a decreased expression of miR-21, increased levels of caspase-3 protein and increased activity of caspase-3. In the TMZ-treated group, the MCapoptosis was interrupted and heart function improved (Fig. 4B-D). These results demonstrate (at least in part) that TMZ improved RV function 

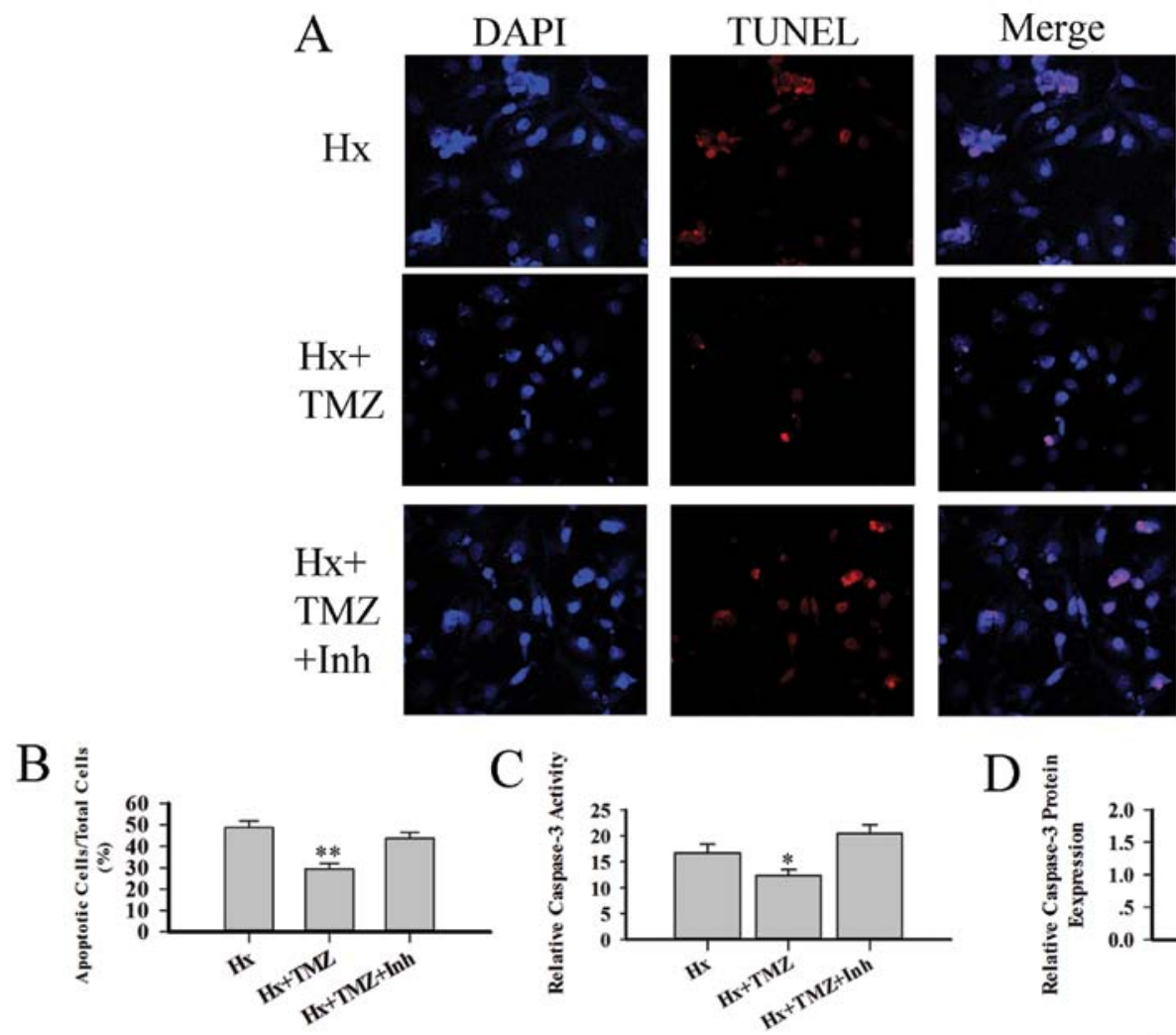

$\mathrm{C} \frac{2}{\frac{2}{2}}$

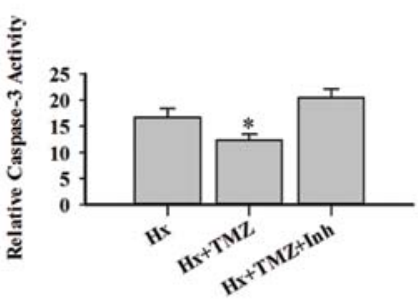

$\mathrm{D} \frac{\sin }{\mathrm{g}}$
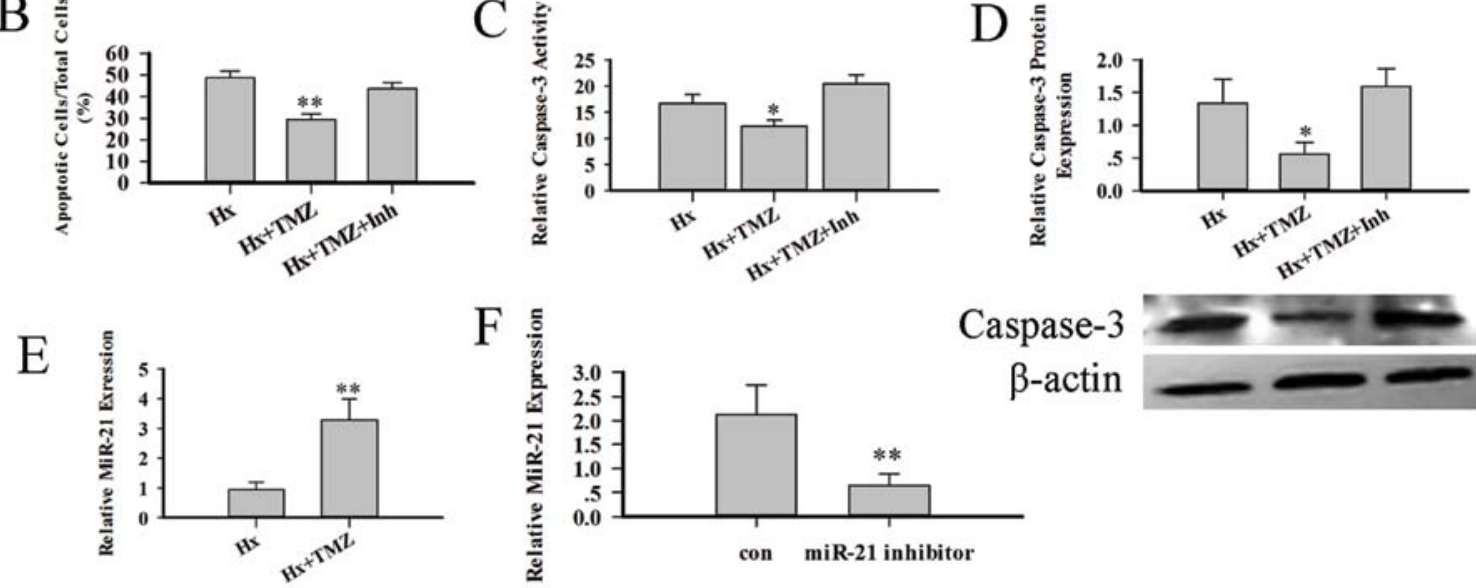

$\mathrm{F}$

Caspase-3

$\beta$-actin

Figure 3. TMZ protects RVMCs against hypoxia-induced apoptosis by regulating miR-21 expression. (A) TUNEL staining was used to evaluate RVMC apoptosis after treatment with TMZ or miR-21 inhibitor. (B) Quantification of TUNEL ${ }^{+}$cells. Data are expressed as percentages. (C) Caspase-3 activity after treatment with TMZ or miR-21 inhibitor. (D) Caspase-3 protein expression after treatment with TMZ or miR-21 inhibitor. (E) Relative expression of miR-21 after TMZ treatment. ${ }^{*} \mathrm{P}<0.05,{ }^{* *} \mathrm{P}<0.01$ compared with the hypoxia group. (F) The miR-21 inhibitor decreased miR-21 expression. ${ }^{* *} \mathrm{P}<0.01$ compared with the control group.
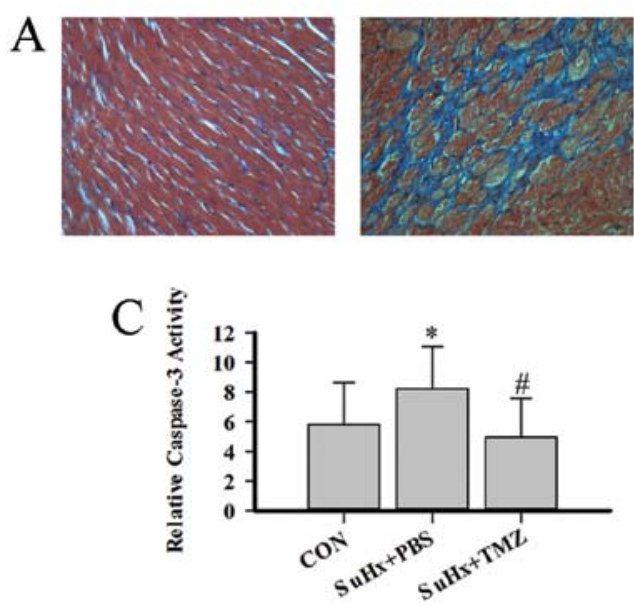

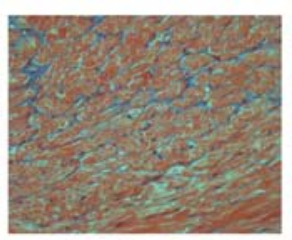

$\mathrm{D}$

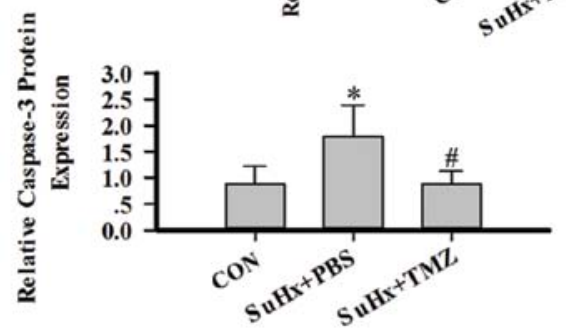

Caspase-3
B

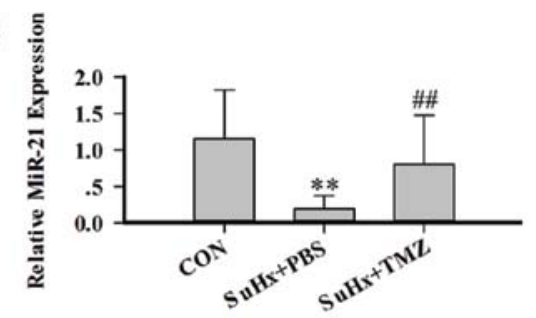

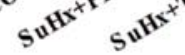

\section{$\beta$-actin}

Figure 4. TMZ improved heart function in rats with right heart failure by increasing miR-21 expression and inhibiting fibrosis. (A) Masson staining used to evaluate fibrosis. (B) qRT-PCR detection used to measure miR-21 expression in isolated RVMCs. (C) Caspase-3 activity assay for the MCs in the control group, SuHx group and SuHx + TMZ group. (D) Caspase-3 protein expression for MCs in the control group, SuHx group and SuHx + TMZ group. "P<0.05, ${ }^{* *} \mathrm{P}<0.01$ compared with the control group, ${ }^{*} \mathrm{P}<0.05$ compared with the SuHx group. 
by increasing miR-21 expression and decreasing the extent of RVMC apoptosis.

\section{Discussion}

The present study demonstrates that the beneficial effect of TMZ on right heart failure and hypoxia-induced apoptosis in RVMCs occurs through an anti-apoptotic function. The anti-apoptotic mechanism is associated with the increased expression of miR-21.

Clinical evidence has shown that the 3-ketoacyl-CoA thiolase (3-KAT) inhibitor, TMZ, improves LV function in elderly patients or those with previous myocardial infarction $(22,23)$. The mechanism of action for this phenomenon is a decrease in the oxidation of fatty acids and stimulation of glucose oxidation (24). These functions directly modify the use of energy substrates by the heart. However, whether this metabolic shift is beneficial or detrimental is controversial. A number of clinical studies have shown that TMZ improves the ejection fraction of individuals with heart failure with or without ischemic cardiomyopathy $(25,26)$. Hence, researchers have begun to pay attention to the non-metabolic functions of TMZ. Liu et al showed that TMZ effectively inhibited myocardial fibrosis through the NADPH oxidase-ROS-CGF signaling pathway (10). The present study shows another non-metabolic effect of TMZ, the inhibition of MC apoptosis. We also demonstrate that the anti-apoptotic function of TMZ os based upon the regulation of miR-21 expression. However, we could not clarify through which pathway TMZ regulates miR-21 expression in RVMCs.

miR-21 is highly expressed in the cardiovascular system, where it regulates the growth and elimination of cardiac cells, as well as the functions of cardiac fibroblasts. Increased miR-21 expression has also been linked to MC hypertrophy (27). Recently, miR-21 was shown to be an important anti-apoptotic factor in MCs under several pathological conditions. miR-21 upregulation has been shown to protect MCs against hypoxiaor $\mathrm{H}_{2} \mathrm{O}_{2}$-induced apoptosis, and phosphatase and tensin homolog (PTEN), FasL and PDCD4 have been identified as targets of miR-21-mediated suppression $(28,29)$. The expression of miR-21 has been shown to be decreased in infarcted regions of the heart, and that the upregulation of miR-21 can decrease the infarcted area (30). The present study also shows the decreased expression of miR-21 in RVMCs after hypoxia. Moreover, we also evaluated miR-21 expression after brief periods of hypoxia (15 $\mathrm{min}$ ) (data not shown). miR-21 expression increased, which was in accordance with the increase observed during ischemic preconditioning (31). During this period, MCs are decompensated. Hence, MCs can directly regulate miR-21 expression, and their own miR-21 expression may be considered to be a regulator of protection. Hence, we speculate that the mechanism by which TMZ regulates miR-21 cannot be separated from its energy optimization role.

We also demonstrate that TMZ improves heart function during right heart failure. Furthermore, such effectiveness may not be due only to energy optimization, but also to antiapoptotic actions and the inhibition of fibrosis. In recent years, a number of studies have shown that RV function is an important predictor of survival in patients with heart failure (32). In 2006, the National Heart, Lung and Blood Institute in the USA identified the function and failure of the RV to be a research priority in cardiovascular disease (33). However, an effective therapy for RV failure is lacking. Clinical research has shown that many effective therapies which apparently improve the prognosis of patients with left heart failure have little merit in those with right heart failure. Animal-based research has shown that MC apoptosis is the major reason for right heart failure. Chronic PH cannot lead to right heart failure, whereas the inhibition of cardiac capillary density with the VEGFR blocker, Su5416, can lead to severe RV failure in rats with RV pressure-overload (19). The present study also demonstrates that, after hypoxia, RVMCs show more severe apoptosis than LVMCs. Hence, we believe that the main issue of right heart failure may be 'running out of fuel', so that the 'hungry' MCs eventually die. Hence, we chose TMZ to treat right heart failure. As expected, TMZ effectively improved RV function, decreased apoptosis and inhibited fibrosis. The results from the present study present strong evidence that TMZ can be used in subjects with right heart failure.

\section{Acknowledgements}

This study was supported in part by the National Basic Research Program (973 Program) of China (grant no. 2007CB512005) and the Key Laboratory of Myocardial Ischemia Mechanism and Treatment (Harbin Medical University), Ministry of Education, China (KF201010/ KF201002).

\section{References}

1. Verzi MP, McCulley DJ, De Val S, et al: The right ventricle, outflow tract, and ventricular septum comprise a restricted expression domain within the secondary/anterior heart field. Dev Biol 287: 134-145, 2005.

2. Zaffran S, Kelly RG, Meilhac SM, et al: Right ventricular myocardium derives from the anterior heart field. Circ Res 95: 261-268, 2004.

3. Haddad F, Hunt SA, Rosenthal DN and Murphy DJ: Right ventricular function in cardiovascular disease, part I: Anatomy, physiology, aging, and functional assessment of the right ventricle. Circulation 117: 1436-1448, 2008.

4. Haddad F, Doyle R, Murphy DJ and Hunt SA: Right ventricular function in cardiovascular disease, part II: pathophysiology, clinical importance, and management of right ventricular failure. Circulation 117: 1717-1731, 2008.

5. Strniskova M, Ravingerova T, Neckar J, et al: Changes in the expression and/or activation of regulatory proteins in rat hearts adapted to chronic hypoxia. Gen Physiol Biophys 25: 25-41, 2006.

6. Wang GY, McCloskey DT, Turcato S, et al: Contrasting inotropic responses to alpha1-adrenergic receptor stimulation in left versus right ventricular myocardium. Am J Physiol Heart Circ Physiol 291: H2013-H2017, 2006.

7. Manchanda SC and Krishnaswami S: Combination treatment with trimetazidine and diltiazem in stable angina pectoris. Heart 78: 353-357, 1997

8. Szwed H, Sadowski Z, Elikowski W, et al: Combination treatment in stable effort angina using trimetazidine and metoprolol: results of a randomized, double-blind, multicentre study (TRIMPOL II). Trimetazidine in POLand. Eur Heart J 22: 2267-2274, 2001.

9. Kober G, Buck T, Sievert H and Vallbracht C: Myocardial protection during percutaneous transluminal coronary angioplasty: effects of trimetazidine. Eur Heart J 13: 1109-1115, 1992.

10. Liu X, Gai Y, Liu F, et al: Trimetazidine inhibits pressure overload-induced cardiac fibrosis through NADPH oxidaseROS-CTGF pathway. Cardiovasc Res 88: 150-158, 2010.

11. Weiss JN, Korge P, Honda HM and Ping P: Role of the mitochondrial permeability transition in myocardial disease. Circ Res 93: 292-301, 2003. 
12. Halestrap AP, Clarke SJ and Javadov SA: Mitochondrial permeability transition pore opening during myocardial reperfusion - a target for cardioprotection. Cardiovasc Res 61: 372-385, 2004.

13. Lim LP, Lau NC, Garrett-Engele P, et al: Microarray analysis shows that some microRNAs downregulate large numbers of target mRNAs. Nature 433: 769-773, 2005.

14. Small EM, Frost RJ and Olson EN: MicroRNAs add a new dimension to cardiovascular disease. Circulation 121: 1022-1032, 2010.

15. Cheng $Y$ and Zhang C: MicroRNA-21 in cardiovascular disease. J Cardiovasc Transl Res 3: 251-255, 2010.

16. da Costa Martins PA and De Windt LJ: MiR-21: A miRaculous Socratic paradox. Cardiovasc Res 87: 397-400, 2010.

17. Ozpolat B, Akar U, Steiner M, et al: Programmed cell death-4 tumor suppressor protein contributes to retinoic acid-induced terminal granulocytic differentiation of human myeloid leukemia cells. Mol Cancer Res 5: 95-108, 2007.

18. Allgayer $\mathrm{H}$ : Pdcd4, a colon cancer prognostic that is regulated by a microRNA. Crit Rev Oncol Hematol 73: 185-191, 2010

19. Bogaard HJ, Natarajan R, Henderson SC, et al: Chronic pulmonary artery pressure elevation is insufficient to explain right heart failure. Circulation 120: 1951-1960, 2009.

20. Kajstura J, Cigola E, Malhotra A, et al: Angiotensin II induces apoptosis of adult ventricular myocytes in vitro. J Mol Cell Cardiol 29: 859-870, 1997.

21. Cacciapuoti F: Echocardiographic evaluation of right heart function and pulmonary vascular bed. Int J Cardiovasc Imaging 25: 689-697, 2009.

22. Vitale C, Wajngaten M, Sposato B, et al: Trimetazidine improves left ventricular function and quality of life in elderly patients with coronary artery disease. Eur Heart J 25: 1814-1821, 2004.

23. Di Napoli P, Taccardi AA and Barsotti A: Long term cardioprotective action of trimetazidine and potential effect on the inflammatory process in patients with ischaemic dilated cardiomyopathy. Heart 91: 161-165, 2005.

24. Kantor PF, Lucien A, Kozak R and Lopaschuk GD: The antianginal drug trimetazidine shifts cardiac energy metabolism from fatty acid oxidation to glucose oxidation by inhibiting mitochondrial long-chain 3-ketoacyl coenzyme A thiolase. Circ Res 86: 580-588, 2000
25. Fragasso G, Palloshi A, Puccetti P, et al: A randomized clinical trial of trimetazidine, a partial free fatty acid oxidation inhibitor, in patients with heart failure. J Am Coll Cardiol 48: 992-998, 2006.

26. Tuunanen H, Engblom E, Naum A, et al: Trimetazidine, a metabolic modulator, has cardiac and extracardiac benefits in idiopathic dilated cardiomyopathy. Circulation 118: 1250-1258, 2008.

27. Tatsuguchi M, Seok HY, Callis TE, et al: Expression of microRNAs is dynamically regulated during cardiomyocyte hypertrophy. J Mol Cell Cardiol 42: 1137-1141, 2007.

28. Sayed D, He M, Hong C, et al: MicroRNA-21 is a downstream effector of AKT that mediates its antiapoptotic effects via suppression of Fas ligand. J Biol Chem 285: 20281-20290, 2010.

29. Cheng Y, Liu X, Zhang S, et al: MicroRNA-21 protects against the $\mathrm{H}(2) \mathrm{O}(2)$-induced injury on cardiac myocytes via its target gene PDCD4. J Mol Cell Cardiol 47: 5-14, 2009.

30. Dong S, Cheng Y, Yang J, et al: MicroRNA expression signature and the role of microRNA-21 in the early phase of acute myocardial infarction. J Biol Chem 284: 29514-29525, 2009.

31. Qian J, Ren X, Wang X, et al: Blockade of Hsp20 phosphorylation exacerbates cardiac ischemia/reperfusion injury by suppressed autophagy and increased cell death. Circ Res 105 1223-1231, 2009.

32. Meyer P, Desai RV, Mujib M, et al: Right ventricular ejection fraction $<20 \%$ is an independent predictor of mortality but not of hospitalization in older systolic heart failure patients. Int J Cardiol 155: 120-125, 2012.

33. Voelkel NF, Quaife RA, Leinwand LA, et al: Right ventricular function and failure: report of a National Heart, Lung, and Blood Institute working group on cellular and molecular mechanisms of right heart failure. Circulation 114: 1883-1891, 2006. 